\title{
Effects of Multi-Joint Angle Changes on EMG Activity and Force of Lower Extremity Muscles during Maximum Isometric Leg Press Exercises
}

\author{
Masako Azegami ${ }^{1}$, RyUya Yanaginashi ${ }^{2}$, KeI MiYoshi $^{3)}$, Katsushi AKahane ${ }^{3)}$, \\ MASAYOSHI OHIRA ${ }^{3)}$, TSUGUTAKE SADOYAMA ${ }^{4)}$ \\ ${ }^{1)}$ Department of Nursing, Shinshu University School of Health Sciences: 3-1-1 Asahi, \\ Matsumoto 390-8621, Japan. TEL \& FAX +81 263-37-2374, \\ E-mail:mazegam@gipac.shinshu-u.ac.jp \\ ${ }^{2)}$ Nagano College of Nursing \\ ${ }^{3)}$ Department of Physical Therapy, Shinshu University School of Health Sciences \\ ${ }^{4}$ Shinshu University School of Textile Science \& Technology
}

\begin{abstract}
The objectives of this study were to identify the position where optimal leg muscle force occurs and to analyze the lower extremity muscle activity by comparing different hip and knee joint angle positions using a newly developed closed kinetic chain (CKC) leg press exercise device. Muscle force and electromyogram (EMG) measurements during maximum voluntary isometric leg presses (MVC-CKC) were performed on 6 healthy women in 6 different knee flexion angles $\left(15^{\circ}, 30^{\circ}, 45^{\circ}, 60^{\circ}, 75^{\circ}, 90^{\circ}\right)$ and 2 different postures (supine and trunk upright posture). Results showed that the muscle force of MVC-CKC reached its peak at $60^{\circ}$ knee flexion angle in both postures. Significant knee angle-dependent differences in EMG activity for both rectus femoris and gastrocnemius muscles were observed, while posturedependent differences in EMG were found in biceps femoris. These particular joint-dependent EMG activities may be explained by the architectural characteristics of bi-articular muscles and muscle lengthtension relationship theory. Co-activation of an antagonist muscle may appear with activation of the gastrocnemius muscle when the knee approaches full extension. Consequently, measurement of isometric $\mathrm{CKC}$ leg extension muscle force at $60^{\circ}$ knee flexion angle in the trunk upright posture seems to be the optimal position.
\end{abstract}

Key words: Closed kinetic chain exercise, Leg extension force, Electromyogram

(This article was submitted Aug. 25, 2006, and was accepted Sep. 28, 2006)

\section{INTRODUCTION}

Previous studies have reported that approximately $20 \%$ of hospitalized elderly patients were confronted with decreased independent activity of daily living (ADL) function due to immobilization or bed rest ${ }^{1)}$. The decline in ADL in elderly individuals is closely related to muscle weakness in their lower extremities ${ }^{2,3)}$. It is well known that bed rest causes a reduction in the muscle mass of the lower extremity, especially in those that are classified as anti-gravity muscles. According to Funato et al., knee extensor and hip flexor muscle strengths decreased by $12 \%$ and $4 \%$ respectively, during a week of bed rest ${ }^{4}$. In addition, it was reported that the muscle force of hip and knee 
extensors among young healthy men recovered by up to only $85-95 \%$ level after 8 weeks following 20 days of bed rest ${ }^{5)}$. These studies suggest the importance of lower extremity muscle training during prolonged periods of bed rest in order to prevent disability resulting from muscle weakness. Recent studies have reported favorable effects on ADL function from lower extremity muscle training in elderly people ${ }^{6,7)}$.

Lower extremity muscle training performed either in the exercise room or at the patient's bedside can be classified into the following 2 categories: open kinetic chain (OKC) and closed kinetic chain $(\mathrm{CKC})$. OKC is defined as exercise where the distal portion of the extremities is able to move freely, with or without any external load, such as chair-seated knee extensions or knee flexions. $\mathrm{OKC}$ is a form of mono-articular exercise, and the exercised muscle is usually isolated, resulting in specific training to that particular muscle ${ }^{8)}$. In contrast, CKC refers to exercise in which the distal portion of the extremities is fixed, as in a squat or a leg press. CKC exercises for lower extremities involve the hip, knee and ankle joints simultaneously. In comparison to $\mathrm{OKC}, \mathrm{CKC}$ exercise has the added advantage of increased proprioceptive feedback by compressive force from the body mass ${ }^{9)}$, the co-activation of agonist and antagonist muscles ${ }^{10-12)}$, and a reduction in anterior tibial shear force during quadriceps contraction ${ }^{13)}$. Therefore, CKC exercise seems to be superior in terms of knee rehabilitation and more ADL-like training. However, CKC exercise devices are generally expensive and bulky and inappropriate for patients on bed rest.

In order to prevent muscle atrophy due to bed rest, we developed a leg press exercise device, which has the capability to measure total leg muscle force as well. In order to achieve optimal leg muscle force and safety during the exercise for patients who are on bed rest, it is necessary to understand and compare leg muscle force and muscle activity in different knee joint angles and postures. Thus, the purpose of this study was to identify the position where optimal leg muscle force occurs and to analyze the lower extremity muscle activity by comparing different hip and knee joint angle positions during an isometric $\mathrm{CKC}$ leg press exercise.

\section{METHODS}

\section{Subjects}

Six eligible women, who were healthy and free of any orthopedic problems, participated as subjects in this study. The mean ( \pm standard deviation) age of the subjects was $30.3( \pm 7.7)$ years old. Subject characteristics are summarized in Table 1. The subjects were fully informed as to the purpose and procedures of the study, and written consent was obtained from all subjects. This research procedure was reviewed and approved by the Medical Ethics Committee of Shinshu University School of Medicine.

\section{Developed leg press device}

The developed leg press device is capable of measuring the leg extension muscle force as well as performing $\mathrm{CKC}$ isotonic and isometric leg press exercises on the bed. The device consists of a steel plate and a core body part (Fig. 1). The core body part is movable horizontally from proximal to distal. The core body part is composed of a plantar pedal where the foot is placed, and a pedal stopper. The pedal is tiltable from $90^{\circ}$ to $0^{\circ}$ at $30^{\circ}$ intervals. The pedal stopper contains a strain gauge that is able to measure the leg extension force. According to the definition of Dillman et al., the developed device can be described as a CKC machine with a moveable boundary (plantar pedal) with an external load (pedal stopper or spring-loaded) ${ }^{8}$. This is similar to a leg press whereby the trunk is fixed and the footplate is mobile. However, in our developed leg press, fixation of the trunk was achieved by using a pair of Velcro straps and rings at the end for gripping and stabilizing the upper extremities (Fig. 2-A, B). In this manner, movement of the trunk upwards during exercise was prevented.

\section{Experimental protocol}

Initially, subjects were asked to perform singlejoint maximum voluntary isometric contractions (MVC) of the quadriceps femoris, hamstrings and gastrocnemius in order to normalize multi-joint isometric leg press EMG activities. MVC of the quadriceps femoris and hamstrings were measured using a torque dynamometer (GT-30, OG Giken). Each subject was seated in a chair with the hip joint angle at $90^{\circ}$, and Velcro straps were applied around the pelvis and thigh to prevent any incidental movements. The torque load receiver was attached 
Table 1. Summary of subject characteristics

\begin{tabular}{ccccc}
\hline Subj. & $\begin{array}{c}\text { Age } \\
(\mathrm{yrs})\end{array}$ & $\begin{array}{c}\text { Height } \\
(\mathrm{cm})\end{array}$ & $\begin{array}{c}\text { Weight } \\
(\mathrm{kg})\end{array}$ & $\begin{array}{c}\text { BMI } \\
\left(\mathrm{kg} / \mathrm{m}^{2}\right)\end{array}$ \\
\hline 1 & 34 & 162 & 53 & 20.2 \\
2 & 34 & 157 & 50 & 20.3 \\
3 & 37 & 165 & 55 & 20.2 \\
4 & 21 & 160 & 45 & 17.6 \\
5 & 20 & 155 & 50 & 20.8 \\
6 & 36 & 155 & 50 & 20.8 \\
\hline Mean & 30.3 & 159 & 51 & 20.0 \\
SD & 7.7 & 4.0 & 3.4 & 1.21 \\
\hline
\end{tabular}

to the left ankle of each subject, and subjects were instructed to perform a maximum voluntary isometric contraction of the knee extensor muscle at $90^{\circ}$ knee flexion. For the MVC measurement of hamstring muscles, the position of the torque load receiver on the left ankle was reversed and subjects were instructed to pull back their lower thigh as hard as possible. Measurements of MVC of ankle plantar flexion were performed using the technique of manual muscle testing. Subjects were given strong verbal encouragement during each MVC measurement. For all the muscles tested, subjects were instructed to perform the contractions twice and to hold for 5 seconds each.

Subsequently, for the multi-joint muscle strength measurements, the subjects were asked to perform two 5-second maximum voluntary isometric leg presses (MVC-CKC) at 6 different knee joint angles $\left(15^{\circ}, 30^{\circ}, 45^{\circ}, 60^{\circ}, 75^{\circ}, 90^{\circ}\right)$ in two different postures (supine and trunk upright posture). The sequence of the testing posture, followed by the sequence for the knee joint angle was randomized before the start of the experiment. Two expert physiotherapists set the knee joint angle of each measurement using a goniometer. In order to prevent the effects of fatigue during testing, a 5minute rest interval was allowed between the supine and trunk upright postures, and at least one-minute rest interval between each knee joint angle.

\section{Surface electromyogram}

The electromyogram (EMG) activity of the left leg was recorded during measurement of MVC and MVC-CKC. After cleaning the skin with alcohol and abrasion paste, $\mathrm{Ag} / \mathrm{AgCl}$ disposable electrodes (Vitrode F, Nihon Kohden) were placed over the muscle bellies of the rectus femoris (RF), biceps

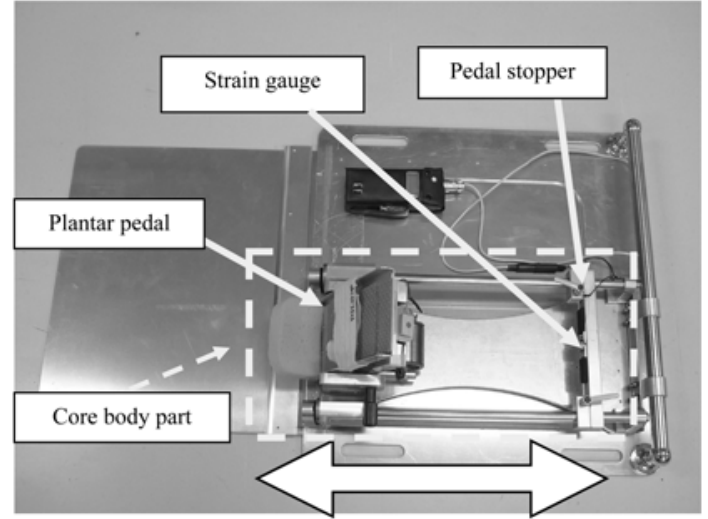

Fig. 1. Leg press device for measurement of isometric leg press force (MVC-CKC). Double-ended arrow indicates the movable range of plantar pedal.

A

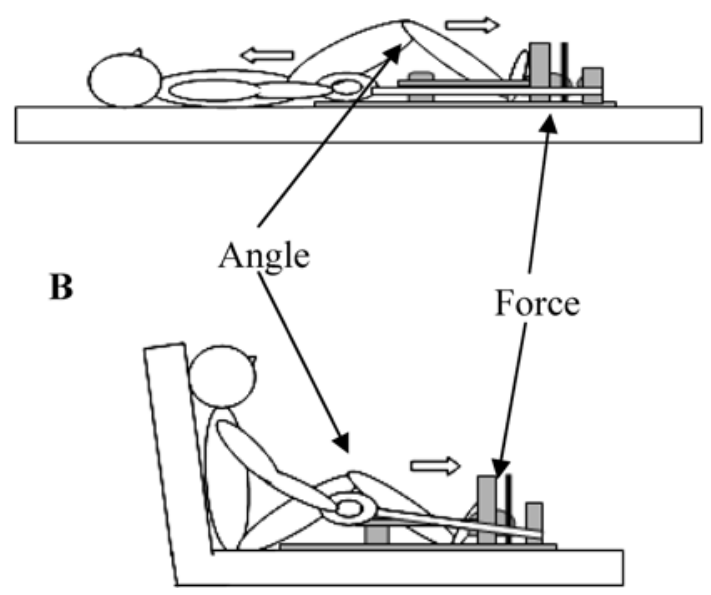

Fig. 2. Schematic diagram of subjects using on the leg press device for measurement of muscle forces (MVC-CKC) during (A) supine, and (B) trunk upright postures.

femoris (BF), and gastrocnemius (GT) muscles with a 2-cm inter-electrode distance. The placement of electrodes was according to the EMG guideline by Basmajian ${ }^{14)}$. After amplification through a differential amplifier (Syna Act MT-11, NEC Medical Systems), the analog EMG signals were converted to digital signals and transmitted to a personal computer at a sampling rate of $1 \mathrm{kHz}$. The stored EMG signals were band-pass filtered at 10 $1,000 \mathrm{~Hz}$ using a multiple bio-amplifier analysis programs (BIMUTAS, Kissei Comtec). From 5 
seconds of recorded data, a 3-second sampling period was used to calculate the integrated EMG (IEMG). Following this, the IEMG values at each joint angle and posture were normalized (\%NIEMG) by dividing it with the MVC values of the corresponding muscle.

\section{Muscle force measurements}

The strain gauge used for the measurement of the muscle force during leg press was calibrated using a digital weighing scale (TBF-102, Tanita), prior to the experiment. The muscle force value during CKC was passed through an analog-digital converter and the peak muscle force values were displayed on a digital transducer indicator with peak hold function (TD-25, Teac), and converted to kilograms based on calibration data and finally recorded manually as MVC-CKC (in kgs). Mean coefficient of variations between the first and second repetitions was in the range of $3.5-5.8 \%$ in the supine, and $5.7-10.5 \%$ in the trunk upright posture.

\section{Statistical analysis}

Descriptive data of \%NIEMG and muscle force are presented as mean and standard error of mean (SEM). Repeated two-way (knee angle by posture) ANOVA and Games-Howell post-hoc test were used to analyze the muscle force and EMG activity and posture-angle relationship. All analyses were performed with the SPSS software (version 11) and a $p$-value of $<0.05$ was considered statistically significant.

\section{RESULTS}

Muscle force-posture - knee joint angle relationship

Four subjects out of 6 MVC-CKC reached peak at $60^{\circ}$ and other 2 subjects reached their peak at $75^{\circ}$ of knee joint angle. The mean peak muscle force occurred at $60^{\circ}$ knee joint flexion in both postures. On the other hand, the mean minimum force value in the supine occurred at $90^{\circ}$, and in the trunk upright posture at $15^{\circ}$. A significant interaction between postures $(\mathrm{F}=142.4, \mathrm{p}<0.01)$ and knee joint angles $(\mathrm{F}=15.7, \mathrm{p}<0.01)$ was observed. Polynomial trend analysis showed that there were significant differences between the two postures from $45^{\circ}$ to $75^{\circ}$ knee flexion angles. Significant differences between adjacent knee angles were observed at $30-45^{\circ}$ and $75-90^{\circ}$ in the trunk upright posture (Table 2).
Table 2. MVC-CKC (in kgf) at each knee flexion angle and posture

\begin{tabular}{ccl}
\hline $\begin{array}{c}\text { Knee flexion } \\
\text { angle (degree) }\end{array}$ & Supine & Trunk upright \\
\hline 15 & $17.8 \pm 0.4$ & $20.2 \pm 0.9$ \\
30 & $18.9 \pm 0.7$ & $22.3 \pm 1.0$ \\
45 & $20.2 \pm 0.6$ & $27.1 \pm 1.0^{* *} \square$ \\
60 & $22.7 \pm 0.7$ & $30.3 \pm 1.7^{*}$ \\
75 & $20.6 \pm 0.9$ & $29.7 \pm 1.8^{*} \square$ \\
90 & $17.7 \pm 1.2$ & $22.7 \pm 1.0$ \\
\hline
\end{tabular}

Data are presented as mean \pm SME.

Significant differences between postures are shown: $* \mathrm{p}<0.05 ; * * \mathrm{p}<0.01$.

Significant differences between adjacent knee joint angles are shown: $\# \mathrm{p}<0.05$.

\section{$\%$ NIEMG-posture-knee joint angle relationship}

Figure 3 shows the representative raw EMG data for the 3 muscles during MVC-CKC exercise.

Rectus femoris $(R F)$ : The \%NIEMG of the RF during MVC-CKC is shown in Table 3. The maximum \%NIEMG was produced at $90^{\circ}$ knee flexion angle in the supine and trunk upright postures. Significant interaction between postures $(\mathrm{F}=16.0, \mathrm{p}<0.05)$ and knee joint angles $(\mathrm{F}=15.3$, $\mathrm{p}<0.05$ ) was observed. Polynomial trend analysis showed the peak \%NIEMG value at $90^{\circ}$ knee flexion angle was significantly greater than those at $15-60^{\circ}$ in the trunk upright posture. In the supine, the peak \%NIEMG value at $90^{\circ}$ knee flexion angle was significantly greater than those at $15^{\circ}$. There was a significant difference in the $\%$ NIEMG activity between the supine and the trunk upright postures only at $15^{\circ}$ knee flexion. Significant differences between adjacent knee angles were observed at $60-75^{\circ}$ in the trunk upright posture.

Biceps femoris $(B F)$ : The \%NIEMG was significantly higher for the trunk upright posture compared with the supine for all knee flexion angles except at $90^{\circ}$ flexion. The peak \%NIEMG value was observed at $15^{\circ}$ knee flexion angle in the trunk upright posture, and at $90^{\circ}$ in the supine. A significant interaction between postures $(\mathrm{F}=55.1$, $\mathrm{p}<0.01)$ was observed. However there was no significant interaction between knee joint angles $(\mathrm{F}=0.73, \mathrm{p}=0.54)$. Polynomial trend analysis results are listed in Table 3.

Gastrocnemius (GT): The peak \%NIEMG value was observed at $30^{\circ}$ knee flexion angle in both the supine and the trunk upright postures. A significant 


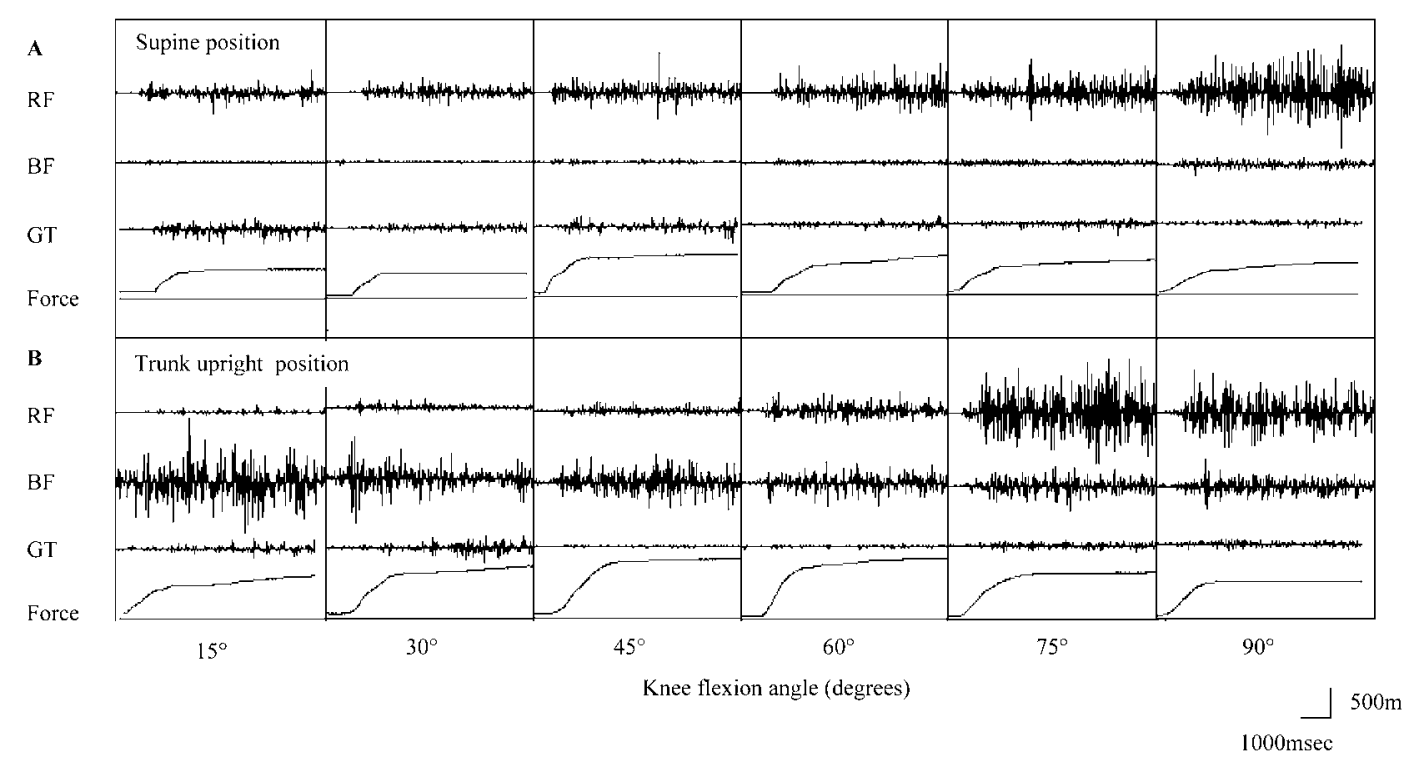

Fig. 3. Raw EMG data (sub No.6) of 3 muscles (RF: rectus femoris, BF: biceps femoris, GT: gastrocnemius) during MVC-CKC in the (A) supine, and (B) trunk upright postures.

interaction between knee joint angles $(\mathrm{F}=17.7$, $\mathrm{p}<0.01$ ) was observed while there was no significant interaction between postures $(\mathrm{F}=1.27, \mathrm{p}=0.28)$. Polynomial trend analysis results are listed in Table 3.

\section{DISCUSSION}

\section{Muscle force and multi-joint angle}

Previous studies, which analyzed the effect of knee joint angle on muscle force during single-joint knee extension (OKC), reported that a peak isometric muscle contraction occurred at approximately $65-80^{\circ}$ of knee flexion ${ }^{15,16)}$, and muscle force was significantly lower when the knee was closer to full extension. Although comparison with other studies is difficult due to different protocol and methodology, knee joint angle and muscle force relationship appear to be similar in isometric multi-joint leg press and single-joint knee extension. In the case of $\mathrm{OKC}$, muscle force generation depends on muscle length, in other words an optimal actin-myosin overlap in sarcomeres from the biomechanical perspective ${ }^{17)}$. In contrast, CKC cannot be explained solely by single muscle length-tension relationship theory because multi-joint contractions induce coactivation of agonist and antagonist muscles possibly to maintain knee stabilization ${ }^{10-13,18)}$.

This study showed that the muscle force of multi- joint isometric leg press reached its peak at $60^{\circ}$ knee flexion angle in both the supine and trunk upright postures. Some studies have investigated the load of knee joint and ligament by comparing knee extension and leg press. Escamilla et al. ${ }^{10)}$ reported that the estimated maximum tibiofemoral and patellofemoral compressive forces were found at $90^{\circ}$ knee flexion angle during dynamic leg press, whereas peak compressive forces during dynamic knee extension were observed at $57^{\circ}$ and $75^{\circ}$ knee flexion angle, respectively. They also found that the leg press and squat at less than $60^{\circ}$ knee flexion angle produced less compressive force compared with OKC. Similar results on the effect of knee flexion angle on compressive stress to the knee were reported from analysis using computer simulation ${ }^{19)}$. These previous studies indicate that CKC exercise at a knee angle of $60^{\circ}$ seems to be relatively safe for preventing exercise-induced injuries from excessive joint stress.

Muscle force measured in the trunk upright posture was greater than those in the supine at all knee joint angles measured in the present study. A possible reason for this difference could be the variation in the stability of the pelvis between the two postures. In the trunk upright posture, the subjects' pelvis was adequately supported by the up-righted bed itself. On the other hand, leg press in the supine produced hip flexion effort of RF that 
Table 3. Relationship between the \%NIEMG activity (in \%) and each knee flexion angle and posture for 3 muscles

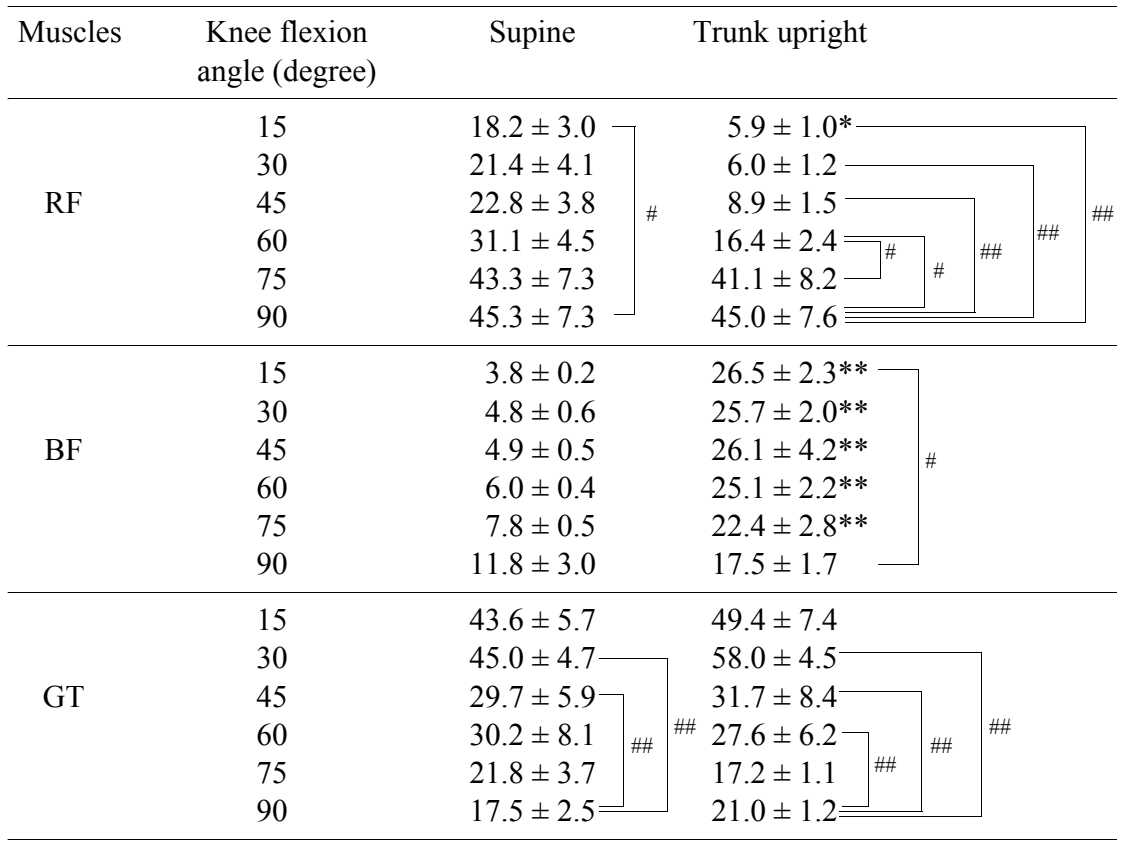

Data are presented as mean $\pm \mathrm{SME}$.

$\mathrm{RF}$, rectus femoris; BF, biceps femoris; GT, gastrocnemius.

Significant differences between postures are shown: ${ }^{*} \mathrm{p}<0.05 ; *{ }^{*} \mathrm{p}<0.01$.

Significant differences between knee joint angles are shown: ${ }^{\#} \mathrm{p}<0.05 ;{ }^{\#} \mathrm{p}<0.01$.

may have led to an anterior tilt of the pelvis, according to the principle of the action-reaction effect that counterbalances the straight downward force of leg press versus the simultaneous load on the upper body.

Consequently, measurement of isometric muscle force at $60^{\circ}$ knee flexion angle seems to be the optimal position since it reduces compressive load to the knee and generates balanced muscle force.

\section{EMG activities and multi-joint angle}

There were significant knee angle-dependent differences in EMG activity for both RF and GT muscles. Meanwhile, EMG activity in BF showed significant posture-dependent differences due to hip joint angle changes. The muscles measured for EMG in this study were all bi-articular muscles, thus knee and hip joint angle changes led to displacement of distal or proximal muscle attachment that resulted in either shortening or lengthening of the muscle ${ }^{20)}$. Thus, specific jointdependent EMG activity could be explained by the architectural perspective in each muscle based on the muscle length-tension relationship theory. Changes of EMG activity patterns by hip and/or knee joint angles in each muscle were consistent with previous reports that examined this relationship under static OKC exercise condition ${ }^{21,22)}$. However, multi-joint angle change of EMG activity during CKC cannot be explained solely by the muscle length-tension theory.

Previous reports have suggested that the coactivation of agonist and antagonist muscles might influence EMG activity during CKC. From the perspective of agonist muscles during CKC, Stensdotter et al. ${ }^{12)}$ explored EMG activity of quadriceps muscles, including RF, vastus medialis and vastus lateralis, during isometric $\mathrm{OKC}$ and $\mathrm{CKC}$ knee extension at $90^{\circ}$ hip flexion and $30^{\circ}$ knee flexion. They found that RF was less active than all vastus muscle groups of the quadriceps in CKC. It was also reported that RF was activated approximately $45 \%$ greater during OKC compared with CKC, whereas EMG activity of vastus medialis and lateralis during CKC were $20 \%$ and $5 \%$ greater, respectively ${ }^{10)}$. Although EMG activity 
of vastus muscle groups was not investigated in the present study, previous reports have indicated that CKC exercise can co-activate other mono-articular muscles and may work in maintaining muscle balance between agonist muscle groups.

The literature also suggests that $\mathrm{CKC}$ exercise might activate antagonist muscles in order to sustain knee stabilization. In particular, the relationship between quadriceps contraction and the anterior cruciate ligament (ACL) has been well documented ${ }^{18,23)}$. Since quadriceps muscle contraction can induce a great amount of stress on the ACL between near full extension of the knee to approximately $60^{\circ}$ of flexion, the higher EMG activity in GT at lower knee joint angles may act as an antagonist muscle ${ }^{18)}$ and help reduce the stress on ACL and tibial anterior shear force ${ }^{10,13)}$. Presumably, therefore, co-activation of antagonist muscle may appear with GT muscle during CKC knee extension exercise.

\section{Clinical applications}

It is clear that $\mathrm{CKC}$ is a more ADL-like exercise than $\mathrm{OKC}$ to simultaneously stimulate co-activation of agonist and antagonist muscles. The outcome of bedside rehabilitation should emphasize not merely muscle strength but also functional improvement of ADL. Thus, the CKC exercise could provide a rehabilitation option for patients to improve functional performance that is more close to ADL while preventing muscle atrophy due to bed rest. The developed CKC exercise device could contribute to the encouragement of lower extremity muscle force measurement, for not only physiotherapists but also other clinical staff, because there is no specific technique for the measurements.

\section{ACKNOWLEDGMENT}

We deeply thank Ah Cheng Goh, RPT, PhD, associate professor at Shinshu University, for his advice and proof reading. This study was supported by Grant-in-Aid for Young Scientists (B: No. 15791293) from the Japan Society for the Promotion of Science (JSPS).

\section{REFERENCES}

1) Gill TM, Allore HG, Holfford TR, et al.: Hospitalization, restricted activity, and the development of disability among older persons. JAMA, 2004, 292: 2115-2124.

2) Guralnik JM, Ferrucci L, Simonsick EM, et al.: Lowerextremity function in persons over the age of 70 years as a predictor of subsequent disability. N Engl J Med, 1995, 332: 556-561.

3) Beissner KL, Collins JE, Holmes H: Muscle force and range of motion as predictors of function in older adults. Phys Ther, 2000, 80: 556-563.

4) Funato K, Matsuo A, Yata H, et al.: Changes in forcevelocity and power output of upper and lower extremity musculature in young subjects following 20 days bed rest. J Gravit Physiol, 1997, 4: S22-S30.

5) Morishima K, Sekiya N, Miyashita S, et al.: Effects of 20 days horizontal bed rest on maintaining upright standing posture in young persons. J Gravit Physiol, 1997, 4: S41-S45.

6) Binder EF, Schechtman KB, Ehsani AA, et al.: Effects of exercise training on frailty in community-dwelling older adults; results of a randomized, controlled trial. J Am Geriatr Soc, 2002, 50: 1921-1928.

7) Reeves ND, Narici MV, Maganaris CN: In vivo human muscle structure and function; adaptations to resistance training in old age. Exp Physiol, 2004, 89: 675-689.

8) Dillman CJ, Murray TA, Hintermeister RA: Biomechanical differences of open and closed chain exercises with respect to the shoulder. JSR, 1994, 3: 228-238.

9) Fritz M: Simulating the response of a standing operator to vibration stress by means of a biomechanical model. J Biomech, 2000, 33: 795-802.

10) Escamilla RF, Fleisig GS, Zheng $\mathrm{N}$, et al.: Biomechanics of the knee during closed kinetic chain and open kinetic chain exercises. Med Sci Sports Exerc, 1998, 30: 556-569.

11) Pincivero DM, Aldworth $C$, Dickerson $T$, et al.: Quadriceps-hamstring EMG activity during functional, closed kinetic chain exercise to fatigue. Eur J Appl Physiol, 2000, 81: 504-509.

12) Stensdotter A-K, Hodges PW, Mellor R, et al.: Quadriceps activation in closed and open kinetic chain exercise. Med Sci Sports Exerc, 2003, 35: 2043-2047.

13) Lutz GE, Palmitier RA, An KN, et al.: Comparison of tibiofemoral joint forces during open-kinetic-chain and closed-kinetic-chain exercises. J Bone Joint Surg Am, 1993, 75: 732-739.

14) Basmajian JV, Blumenstein R: Electrode Placement in EMG Biofeedback. Baltimore: Williams \& Wilkins, 1980, pp 81-87.

15) Pincivero DM, Salfetnikov Y, Campy RM, et al.: Angle- and gender-specific quadriceps femoris muscle recruitment and knee extensor torque. J Biomech, 2004, 37: 1689-1697.

16) Welsch MA, Williams PA, Pollock ML, et al.: Quantification of full-range-of-motion unilateral and bilateral knee flexion and extension torque ratios. Arch Phys Med Rehabil, 1998, 79: 971-978. 
17) Rassier DE, MacIntosh BR, Herzog W, et al.: Length dependence of active force production in skeletal muscle. J Appl Physiol, 1999, 86: 1445-1457.

18) Fleming BC, Renstrom PA, Ohlen G, et al.: The gastrocnemius muscle is an antagonist of the anterior cruciate ligament. J Orthop Res, 2001, 19: 1178-1184.

19) Cohen ZA, Roglic H, Grelsamer RP, et al.: Patellofemoral stresses during open and closed kinetic chain exercises. Am J Sports Med, 2001, 29: 480-487.

20) Neumann DA: Kinesiology of the Musculoskeletal System. In: Foundations for Physical Rehabilitation.
St. Louis: Mosby, 2002, pp 387-476.

21) Maffiuletti NA, Lepers R: Quadriceps femoris torque and EMG activity in seated versus supine position. Med Sci Sports Exerc, 2003, 35: 1511-1516.

22) Pavol MJ, Grabiner MD: Knee strength variability between individuals across ranges of motion and hip angles. Med Sci Sports Exerc, 2000, 32: 985-992.

23) Fitzgerald GK: Open versus closed kinetic chain exercise: issues in rehabilitation after anterior cruciate ligament reconstructive surgery. Phys Ther, 1997, 77: 1747-1754. 